OPEN ACCESS

Edited by:

Ke Liu,

Capital Medical University, China

Reviewed by:

Paul Van De Heyning,

University of Antwerp, Belgium

Samira Anderson,

University of Maryland, College Park,

United States

*Correspondence:

Xinxing Fu

xinxing.fu@research.uwa.edu.au

Bo Liu

trliubo@139.com

Shuo Wang

shannonwsh@aliyun.com

Specialty section:

This article was submitted to Auditory Cognitive Neuroscience,

a section of the journal

Frontiers in Neuroscience

Received: 29 July 2021

Accepted: 22 October 2021

Published: 26 November 2021

Citation:

Fu X, Liu B, Wang S, Eikelboom RH and Jayakody DMP (2021) The Relationship Between

Hearing Loss and Cognitive Impairment in a Chinese Elderly Population: The Baseline Analysis.

Front. Neurosci. 15:749273. doi: 10.3389/fnins.2021.749273

\section{The Relationship Between Hearing Loss and Cognitive Impairment in a Chinese Elderly Population: The Baseline Analysis}

\author{
Xinxing Fu' ${ }^{1,2,3 *}$, Bo Liu' ${ }^{1 *}$, Shuo Wang ${ }^{1 *}$, Robert H. Eikelboom ${ }^{2,3,4}$ and \\ Dona M. P. Jayakody 2,3,5
}

\begin{abstract}
1 Beijing Institute of Otolaryngology, Otolaryngology-Head and Neck Surgery, Beijing Tongren Hospital, Capital Medical University, Beijing, China, ${ }^{2}$ Medical School, The University of Western Australia, Crawley, WA, Australia, ${ }^{3}$ Ear Science Institute Australia, Subiaco, WA, Australia, ${ }^{4}$ Department of Speech Language Pathology and Audiology, University of Pretoria, Pretoria, South Africa, ${ }^{5}$ WA Centre for Health and Ageing, The University of Western Australia, Crawley, WA,
\end{abstract} Australia

Objectives: The objective of the study was to investigate the association between untreated age-related hearing loss and cognitive impairment in Mandarin-speaking older adults living in China.

Methods: Older adults (293; 111 males, $M=70.33 \pm 4.90$ years; 182 females, $M=69.02 \pm 4.08$ years) were recruited. All participants completed a pure tone audiometric hearing assessment, Hearing Impairment-Montreal Cognitive Assessment Test (HI-MoCA), and a computerized neuropsychology test battery (CANTAB). The Mandarin version of the De Jong Gierveld Loneliness Scale was used to measure the loneliness, and the Mandarin version of the 21-item Depression Anxiety Stress Scale (DASS-21) was used to measure the current severity of a range of symptoms common to depression, stress, and anxiety of the participants.

Results: A multiple stepwise regression analysis showed that the average of four mid-frequency thresholds in the better ear was related to CANTAB Paired Associates Learning $(\beta=0.20, p=0.002)$, and the global cognitive function score (HI-MoCA) $(\beta=-0.25, p<0.001)$. The average of three high frequencies in the better ear was significantly associated with CANTAB Delayed Matching to Sample ( $\beta=-0.16$, $p=0.008)$, and Spatial Working Memory $(\beta=0.17, p=0.003)$.

Conclusion: The results revealed a significant relationship between age-related hearing loss and cognitive impairment in Mandarin-speaking older adults. These research outcomes have clinical implications specifically for hearing health care professionals in China and other populations that speak a tonal language, especially when providing hearing rehabilitation.

Keywords: age-related hearing loss (ARHL), cognitive impairment, depression, loneliness, tonal language 


\section{INTRODUCTION}

An estimated 1.57 billion people worldwide had hearing loss in 2019, accounting for $20.3 \%$ of the global population (Haile et al., 2021). Approximately one-third of people over 65 years of age are affected by disabling hearing loss. Hearing loss hampers effective communication, which can have a significant impact on the activities of daily life causing feelings of loneliness, isolation, and frustration (Ciorba et al., 2012). Depression, anxiety, and stress are also associated with untreated hearing loss (Jayakody et al., 2018a).

Studies conducted with non-tonal language speakers have established a relationship between untreated age-related hearing loss and cognitive impairment and dementia (Deal et al., 2015; Jayakody et al., 2018b). Those with mild, moderate, and severe hearing loss are two to five times more likely to develop dementia compared with those with normal hearing sensitivity (Lin et al., 2011).

Approximately $50 \%$ of the population of the world speaks a tonal language, e.g., Mandarin, Cantonese. In tonal languages, changes in pitch (tone) at the monosyllabic level convey lexical meaning of a word. As pitch is the important factor here, tonal language perception can be equated with music perception. This view is supported by evidence that speaking tonal language leads to enhanced pitch perception in music. The benefits of music training and tonal language experience are bidirectional, and a background in either domain improves processing in the other domain (Ngo et al., 2016). Furthermore, playing a musical instrument may have the benefits on slowing agerelated cognitive decline (Balbag et al., 2014). This may be explained by the fact that playing a musical instrument trains executive function involving different cognitive functions of the brain, including memory, visual, and executive functions (Mansky et al., 2020).

There is also psychophysiological evidence that suggests that a tonal language background maybe associated with enhanced general cognitive abilities. A study conducted by using the Corsi blocks tapping test has shown that the Cantonese speakers had a superior working memory related to pitch perception relative to English speakers (Bidelman et al., 2013).

Therefore, it is possible to hypothesize that tonal language speakers are likely to have better working memory in their later life compared with non-tonal language older adults. As hearing sensitivity is strongly associated with age, this raises the question whether speaking a tonal language is a protective factor for cognitive decline in older adults.

There are a handful of studies that have investigated the associations between cognitive function and untreated hearing loss in tonal language speakers. However, these studies have reported conflicting findings. Luo et al. (2018) reported that the prevalence of dementia was $0.61(95 \% \mathrm{CI}=0.53-0.71 \%)$ with hearing impairment, whereas Gyanwali et al. (2020) failed to find an independent association between hearing loss and dementia $(p>0.05)$.

An analysis of the cognitive assessments utilized in studies that investigated the association between hearing loss and cognitive impairment/dementia in tonal language speakers have not thoroughly assessed cognition, or often used verbally loaded test materials that rely on audition, i.e., some of the materials are presented orally-aurally. For example, a population-based study of age-related hearing loss and dementia in Taiwan was only able to assess the cognitive status of the participants as impaired or normal by psychiatric diagnosis, not enabling a full quantitative analysis (Su et al., 2017). A cohort study investigated the association between hearing loss with cognitive function in a Han Chinese cohort using a standardized neurocognitive battery (Ren et al., 2019). However, as both of these studies used verbal language instructions, it is possible that the hearing loss of the participants had an impact on their cognitive performance, causing an overestimate on the degree of cognition impairment (Dupuis et al., 2015).

The underlying mechanism between hearing loss and cognitive impairment is yet to be established. However, the posited underlying factors of this mechanism may include low education, hypertension, obesity, smoking, depression, physical inactivity, and low social contact (Livingston et al., 2017).

Therefore, this study explored the potential association between hearing loss and cognitive impairment in Mandarinspeaking older adults in China using a non-verbal cognitive assessment test battery, controlling for a number of potential confounders.

\section{MATERIALS AND METHODS}

Participants were recruited through social media advertisements, flyers, and community centers in Beijing. Ethics approval for this study was obtained from The University of Western AustraliaHuman Research Ethics Committee (RA/4/20/5538) and Beijing Tongren Hospital, Capital Medical University (TRECKY2019090). All procedures were carried out in accordance with these approvals, and the participants all provided written informed consent.

Inclusion criteria: Native Mandarin speakers, aged 60 years and above with hearing impairment were invited to participate in the study. Exclusion criteria: Those not in general state of good health or unable to perform tasks required in the cognition evaluation session due to an underlying physical or mental condition, or those who had previously worn or currently wearing hearing aids or a hearing implant were excluded from the study. Those who could not complete the Motor Screening Task (MOT) module of the Cambridge Neuropsychological Test Battery (CANTAB) (Cambridge Cognition Ltd., 2014) due to inability to follow instructions, or dexterity problems were also excluded from the study.

\section{Measure and Procedure}

The assessment methods comprised measurements of hearing ability, cognition function, loneliness, and mental health status.

After enrollment, all participants completed a baseline questionnaire capturing demographic information, and data on alcohol consumption (never, less than 14, 15-28, 29-42, or 43 or more standard drinks per week), smoking (never, past, current, or exposed to second-hand smoking), self-reported chronic disease 
history (heart disease, stroke, high cholesterol, atherosclerosis, hypertension, diabetes, frequent childhood ear infections, trauma to ear or head, and depression), leisure activities, as well as marital status and living arrangements. Leisure activities were classified into recreational, intellectual, physical, and social categories (Leung et al., 2010). Living arrangements were recorded as living with spouse, living with children, or living alone, and marital status as single, married, widowed, or divorced.

\section{Hearing Assessment}

Pure-tone audiometry (PTA) was assessed with an audiometer (Conera Audiometer, GN Otometrics Ltd., Denmark) and supraaural earphone (TDH-39). For all participants, bilateral airconduction thresholds were measured at $0.25,0.5,1,2,4,6$, and $8 \mathrm{kHz}$; and bone-conduction thresholds were measured at $0.5,1,2$, and $4 \mathrm{kHz}$, through standard audiometric assessment conducted by a qualified audiologist in a soundproof booth at the audiology center of the Beijing Institute of Otolaryngology.

Evaluation of hearing loss was deployed by two different methods: the traditional four frequency average of hearing thresholds at $0.5,1,2$, and $4 \mathrm{kHz}$, and the three high frequency average of hearing thresholds at 4,6 , and $8 \mathrm{kHz}$ in the better ear, noted, respectively, as 4FA and 3HFA. These were analyzed as continuous variables. For the purpose of summarizing the hearing loss of the study cohort, the 4FA hearing loss was classified using the World Health Organization (WHO) grades of hearing impairment (Humes, 2019), respectively, normal hearing-less than $20 \mathrm{~dB} \mathrm{HL}$; mild hearing loss-20 to $<35 \mathrm{~dB}$ $\mathrm{HL}$; moderate hearing loss -35 to $<50 \mathrm{~dB} \mathrm{HL}$; moderately severe hearing loss-50 to $<65 \mathrm{~dB} \mathrm{HL}$; severe hearing loss65 to $<80 \mathrm{~dB} \mathrm{HL}$; profound hearing loss -80 to $<95 \mathrm{~dB} \mathrm{HL}$; complete hearing loss-95 dB HL or greater in the better ear.

\section{Cognitive Assessment}

The global cognitive functions of the subjects were tested using the Montreal Cognitive Assessment Test (MoCA) for the hearing impaired. A Mandarin version of the MoCA (version 7.2) was adapted (Nasreddine et al., 2005), which was converted into a timed PowerPoint (Microsoft Corp., Redmond, Washington, United States) presentation, here named HI-MoCA (Hearing impaired-MoCA), with all the verbal instructions replaced with visual instructions (Lin et al., 2017).

For the visuospatial and executive tasks, an answer sheet was given to the subject to be completed as instructed. For all other tasks, the subjects were asked to verbally answer or respond. For example, for the memory recall section, a series of words were presented at a rate of $2 \mathrm{~s}$ per word (truck, banana, violin, desk, and green), comparable to the standard verbal administration of the original MoCA. In the attention tasks, for both the forward and backward order digits repetition, all the numbers were presented at a rate of $1.5 \mathrm{~s}$ (compared with one digit per second in the original MoCA). For the digits series task, requiring the participant to identify the digit 1 among a string of digits from 0 to 9 by tapping the table, the HI-MoCA visually presented a series of numbers at a rate of $1.5 \mathrm{~s}$ between numbers for the subject to observe and respond to by tapping the table. In the language task, both sentences are presented for $9 \mathrm{~s}$.
All the HI-MoCA tests were administered by an audiologist, who received formal training from a senior neurology physician with advanced clinical experience and completed the certificated training course via the MoCA website.

Non-verbal cognition was assessed using the Cambridge Neuropsychological Test Automated Battery (CANTAB) (Campos-Magdaleno et al., 2020). The CANTAB software was installed on a laptop with an integrated touch screen (IBM, Yoga S, with Windows 10.1 platform).

Evaluation of cognitive function for this study was focused on working and episode memory, processing speed, and spatial information processing. Therefore, the relevant test items were chosen from the CANTAB test battery, including delayed matching to sample (DMS), paired associates learning (PAL), and spatial working memory (SWM), respectively. Assessment commenced with a test of motor function, to qualify the participant for further assessment.

\section{Motor Screening Task}

The motor screening task provides a general assessment of whether vision, sensorimotor deficits, or lack of comprehension, which, if present, would limit the collection of valid data from the participant (Cambridge Cognition Ltd., 2014). It involved the selection of colored crosses in different positions on the screen as quickly and accurately as possible by the participant. MST outcome measures assess the speed of response and the accuracy of pointing of the participant.

\section{Delayed Matching to Sample}

The DMS test assesses both simultaneous visual matching ability and short-term visual recognition memory, for non-verbalizable patterns (Cambridge Cognition Ltd., 2014). DMS is primarily sensitive to damage in the medial temporal lobe area, with some input from the frontal lobes (Olsen et al., 2009). The participant is shown a complex visual pattern (the sample) and four response patterns. The task of the participant is to identify the response pattern that is identical to the sample pattern. Response patterns will be shown simultaneously with the sample pattern, whereas in others, a delay (of 0,4 , or $12 \mathrm{~s}$ ) is introduced. DMS outcome measures include latency (the speed of response of the participant), the percentage of correct patterns selected, and a statistical measure giving the probability of an error after a correct or incorrect response. The DMS parallel mode was administered for all the subjects, including three practice trials and 20 assessment trials. The average administration time is around $8 \mathrm{~min}$. Percentage of correct patterns selected by the participant was analyzed in this study (Cambridge Cognition Ltd., 2014).

\section{Paired Associates Learning}

The PAL test assesses episodic visuospatial memory, learning, and association ability (Cambridge Cognition Ltd., 2014). This test is primarily sensitive to changes in medial temporal lobe functioning (Cambridge Cognition Ltd., 2014). In this task, six to eight white boxes are displayed on a computer screen. These briefly reveal a pattern which varies in shape and color. The task of the participant is to remember the pattern revealed and 
match it to the pattern that appears in the middle of the screen by touching the box that contains the correct pattern. The task is made progressively difficult by presenting 1,2,3,6, and 8 patterns to the participant. Outcome measures include the errors made by the participant, the number of trials required to locate the pattern(s) correctly, memory scores, and stages completed. The PAL clinical mode was administered for all the subjects with up to eight stages depending on the performance of the subjects; each stage may have up to 10 trials (attempts) in total. The average administration time is around $10 \mathrm{~min}$ but is greatly affected by the degree of impairment of the subject and the number of repeat presentations that are required. PAL errors (total shapes) and PAL errors (six shapes) were analyzed in this study (Cambridge Cognition Ltd., 2014).

\section{Spatial Working Memory}

The SWM test requires retention and manipulation of visuospatial information. This self-ordered test has obvious executive function demands and provides a measure of nonverbal working memory, visuospatial working memory, and strategy use (Cambridge Cognition Ltd., 2014). SWM is a sensitive measure of frontal lobe and "executive" dysfunction. The task of the participant was to locate tokens hidden in increasing number of boxes $(3,4,6$, and 8$)$. Each box contained only one token per sequence. Searching a box more than once during a sequence results in a "within search error" and revisiting a box in which a token has been found before incurs a "between search error." A "strategy" score, calculated for the more difficult six- and eight-box levels, represented the use of an efficient strategy based on predetermined sequence. Poor use of strategy is reflected in higher strategy scores and vice versa. Three main outcome measures, spatial working memory within errors, between errors, and strategy scores, were calculated for the purpose of this study. The SWM clinical mode was administered for all the subjects with 16 stages, including four practices and 12 assessment trials. The average administration time is around $9 \mathrm{~min}$ but is also affected by the degree of impairment of the subjects.

\section{Loneliness Measurement}

The Mandarin version of the six-item De Jong Gierveld Loneliness Scale was used to measure the loneliness of the subjects (Leung et al., 2008; Yang et al., 2018; Fung et al., 2019). In this six-item scale, three statements are made about emotional loneliness and three about social loneliness. There are negatively (items 1, 2, and 3) and positively (items 4, 5, and 6) worded items, scored 1 point for "Yes" and "More or less" answers, and 0 points for "No" answers to questions 1-3; scored 1 point for "More or less" and "No" answers, and 0 points for "Yes" answers to questions 4-6.

\section{Assessment of Depression, Anxiety, and Stress}

The Depression Anxiety Stress Scales (DASS-21) is a questionnaire to assess the symptoms of depression, anxiety, and stress (Chan et al., 2012; Wang K. et al., 2016). The 21 items on the questionnaire comprise a set of three self-reported scales designed to assess depression, anxiety, and stress status of subjects. The seven elements on each scale are graded on a Likert scale from 0 to 3 (0: "Did not apply to me at all," 1: "Applied to me to some degree or some of the time," 2: "Applied to me to a considerable degree or a good part of the time," and 3: "Applied to me very much or most of the time"). Depression, anxiety, and stress scores are determined by summarizing the scores of the related items (Lovibond and Lovibond, 1995). As the DASS-21 is a shorter version of the 42-item original DASS, the score for each subscale is multiplied by two to calculate the final score.

\section{Statistics Analysis}

All statistical analyses were performed using SPSS version 25 (SPSS Inc., Chicago, IL, United States). A multiple forward stepwise regression analysis was used to examine the relationship between cognitive functions and other variables, including age, gender, smoking and alcohol consumption, chronic medical history, education years, loneliness and mental health, and PTA thresholds of better ear average across $500 \mathrm{~Hz}-8 \mathrm{kHz}$. The different CANTAB modules and HI-MoCA scores were entered as dependent variables, respectively. The collinearity tests were examined during each stepwise regression analysis.

At each step of the regression analysis, variables were added based on $p$-values, and the Akaike Information Criterion (AIC) was used to set a limit on the total number of variables included in the final model. The criteria of entry of each variable was a probability of $F \leq 0.05$ and removal of each variable a probability of $F \geq 0.10$.

\section{RESULTS}

\section{Descriptive Data}

The demographic information, cognitive function scores, and other descriptive data of this study population are presented in Table 1, including the age, gender, education years, hearing threshold, smoking status, alcohol intake, history of chronic disease, depression, anxiety, and stress status of the participants. A total of 293 Mandarin speakers aged between 60 and 87 years were included in the study. The study cohort consisted of 111 males (mean age $=70.33 \pm 4.90$ years) and 182 females (mean age $=69.02 \pm 4.08$ years) .

For descriptive purposes, the better ear 4FA was used to group the participants; 65 had normal hearing, 106 had mild hearing loss, 54 had moderate hearing loss, 34 had moderately severe hearing loss, 31 had severe hearing loss, and three had profound hearing loss, as shown in Table 1.

\section{Regression Analysis}

The multiple stepwise regression analyses revealed that the better ear 4FA significantly predicted HI-MoCA scores $(p<0.001)$, PAL error for 6 shapes $(p<0.05)$, and that the better ear 3HFA significantly predicted SWM within error $(p<0.05)$ and DMS correct percent $(p<0.05)$. The detailed results are shown in Table 2. Only the last model of the stepwise regression is shown. Each model of forward stepwise regression for HI-MoCA, SWM 
between error, SWM within error, SWM strategy, PAL error (all shapes), PAL error (six shapes), DMS correct percent are, respectively, shown in Supplementary Tables 1-7.

\section{DISCUSSION}

This study investigated the association between hearing loss and global cognitive abilities (HI-MoCA) and multiple domains of non-verbal cognitive abilities in Mandarin-speaking older adults. To the best of our knowledge, this is the first study to elucidate the association between hearing loss and cognitive function by using the hearing impairment version MoCA and a non-verbal neurocognitive assessment tool with tonal language participants.

Our results are consistent with previous cross-sectional studies of pure-tone audiometric hearing loss and cognitive decline and impairment in non-tonal language speakers (Gussekloo et al., 2005; Deal et al., 2015; Jayakody et al., 2018b). Our results revealed that both mid-frequency hearing sensitivity and selfreported hearing loss were significantly associated with HIMoCA score.

As shown in Table 2, self-reported hearing loss was significantly associated with both HI-MoCA $(\beta=0.18, p=0.004)$ and PAL error (six shapes) $(\beta=-0.16, p=0.009)$. This finding suggests that self-reported hearing loss may be a useful indicator when evaluating the relationship between hearing loss and cognitive impairment. This finding accords with another study, which showed that self-reported hearing loss was significantly associated with both baseline and follow-up cognitive impairment (Amieva et al., 2015).

Our results also showed that the age-related hearing loss significantly associated with poor performance on some of the cognitive domains, specifically DMS (short-term visual memory) and PAL (episodic visuospatial memory). Prior studies have noted that episodic memory impairments are one of the earliest signs of amnestic mild cognitive impairment, Alzheimer's disease, and other dementias (Wang P. et al., 2016).

Short-term visual memory function in this study was assessed using DMS module of the CANTAB test battery. Linear regression showed that while high-frequency hearing in the better ear was significantly associated with the DMS score, mid-frequency hearing was not associated with the DMS score. This finding is consistent with previous research (Jayakody et al., 2018b) on non-tonal language speakers. A possible explanation for this is that presbycusis usually begins with reduced hearing sensitivity in the higher frequencies while remaining relatively better in the low and mid-frequency range. Consequently, the association between DMS and mid-frequency hearing impairment was not observed in the regression analysis.

Visual memory and learning ability were measured by PAL task of CANTAB test battery. There was a significant positive correlation between mid-frequency hearing in (4FA) in the better ear, and PAL error (six shapes). Previous studies have also demonstrated that performance on the PAL task is highly associated with cognition impairment, and can differentiate subjects with Alzheimer's disease from normal controls with high sensitivity and specificity (Hicks et al., 2020). However, no
TABLE 1 | Descriptive data of the participants.

\begin{tabular}{|c|c|}
\hline Characteristics & All subjects \\
\hline & $N=293$ \\
\hline Male sex, no. (\%) & $111(37.9 \%)$ \\
\hline Smoking, no. (\%) & $107(36.5 \%)$ \\
\hline Alcohol consumption ${ }^{\mathrm{a}}$, no. (\%) & $279(95.2 \%)$ \\
\hline Vascular disease ${ }^{\mathrm{b}}$, no. (\%) & $231(78.8 \%)$ \\
\hline Diabetes, no. (\%) & $66(22.5 \%)$ \\
\hline Frequent childhood ear infections, no. (\%) & $13(4.4 \%)$ \\
\hline Depression clinically diagnosed, no. (\%) & $10(3.4)$ \\
\hline Self-report HL, no. (\%) & $212(72.4 \%)$ \\
\hline Noise exposure, no. (\%) & $52(17.7 \%)$ \\
\hline Living alone, no. (\%) & $17(5.8 \%)$ \\
\hline Single or divorced, no. (\%) & $33(11.3 \%)$ \\
\hline Age (years) & $69.52 \pm 4.45$ \\
\hline Education (years) & $12.98 \pm 2.84$ \\
\hline \multicolumn{2}{|l|}{ Hearing loss classification, no. (\%) } \\
\hline Normal hearing & $65(22.2 \%)$ \\
\hline Mild hearing loss & $106(36.2 \%)$ \\
\hline Moderate hearing loss & $54(18.4 \%)$ \\
\hline Moderately severe hearing loss & $34(11.6 \%)$ \\
\hline Severe hearing loss & $31(10.6 \%)$ \\
\hline Profound hearing loss & $3(1.0 \%)$ \\
\hline 4FA (dB HL) & $36.94 \pm 18.78$ \\
\hline 3HFA (dB HL) & $52.31 \pm 20.17$ \\
\hline Emotional loneliness & $0.92 \pm 0.84$ \\
\hline Social loneliness & $1.11 \pm 1.18$ \\
\hline Loneliness & $2.03 \pm 1.59$ \\
\hline Depression & $3.65 \pm 5.22$ \\
\hline Anxiety & $5.98 \pm 5.58$ \\
\hline Stress & $6.45 \pm 6.70$ \\
\hline Recreational activities score & $14.5 \pm 10.68$ \\
\hline Intellectual activities score & $20.87 \pm 12.72$ \\
\hline Physical activities score & $21.15 \pm 11.06$ \\
\hline Social activities score & $2.11 \pm 2.79$ \\
\hline HI-MoCA score & $25.00 \pm 2.63$ \\
\hline SWM between error & $35.99 \pm 16.25$ \\
\hline SWM within error & $6.83 \pm 5.49$ \\
\hline SWM strategy & $34.64 \pm 5.21$ \\
\hline PAL error (all shapes) & $32.54 \pm 26.66$ \\
\hline PAL error (six shapes) & $7.60 \pm 7.66$ \\
\hline DMS correct percent & $79.43 \pm 11.75$ \\
\hline
\end{tabular}

The data are presented as means \pm standard deviations, unless otherwise indicated.

4FA: four frequencies (500 Hz, 1, 2, and $4 \mathrm{kHz}$ ) average of pure tone hearing thresholds of the better ear; 3HFA: three high frequencies (4, 6, and $8 \mathrm{kHz}$ ) average of pure tone hearing thresholds of the better ear; HI-MoCA, hearing impairedMontreal cognitive assessment test; PAL, paired associates learning; DMS, delayed matching to sample; SWM, spatial working memory.

${ }^{a}$ Alcohol consumption reference is never or less than 14 standard drinks per week. ${ }^{b}$ Vascular disease indicates any one of heart disease, stroke, high cholesterol, atherosclerosis, or hypertension.

significant association was found between PAL scores (all shapes) and hearing loss.

Two types of leisure activities (intelligent and social) were observed in the regression model to be associated with PAL error (all shapes), but not PAL error (6 shapes). Prior studies that have noted the importance of participation in leisure activities, which 
has been associated with the reduced risk of cognitive impairment after controlling for age, gender, and education (Verghese et al., 2003). In this study, information regarding four categories of leisure activities was collected by a questionnaire. It is difficult to explain this result that why PAL error (all shapes) and PAL error (six shapes) showed different regression outcomes, but it may be related to the high level of difficultly of the PAL eight shapes task. Participants sometimes took a very long time on the task and lost their patience, and the results are likely to be inaccurate and unreliable.

Spatial working memory requires retention and manipulation of visuospatial information. The SWM task has notable demands on executive function and provides a measure of strategy as well as working memory. This study showed that high frequency hearing is significantly associated with SWM within error, but not with mid-frequency hearing. This result may be explained, as noted earlier, by the fact that high frequencies show a decrease in sensitivity at least a decade earlier than for the mid-frequency speech frequencies (Salvi et al., 2018). However, neither 4HA nor 3HFA were associated with SWM between error and SWM strategy. This finding is contrary to a previous study which has suggested that both the SWM errors and strategy score are associated with mid-frequency hearing (Jayakody et al., 2018b).

Education was significantly associated with almost all the cognitive-dependent variables, including HI-MoCA and CANTAB DMS, PAL, and SWM. These results corroborate the findings of a great deal of the previous work in this field. Lower rates of late-life dementia are associated with higher education levels during earlier life (Livingston et al., 2017). Early education may promote the development during a sensitive period of childhood that protect against late-life cognitive decline (Zahodne et al., 2015).

Another important finding was that social loneliness and overall loneliness (social and emotional) were significantly associated with both SWM between error and SWM strategy. Several reports have shown that both loneliness and social isolation are associated with cognitive decline (Donovan et al., 2017; Lara et al., 2019). The literature does not testify the causal relationship between loneliness and cognitive function. Loneliness may lead to cognitive decline, perhaps from limited social interactions, but it is also possible that cognitive impairment may lead to limited social interactions and further loneliness (Boss et al., 2015).

TABLE 2 | Multiple stepwise regression between CANTAB/HI-MoCA scores and other variables.

\begin{tabular}{|c|c|c|c|c|c|c|c|c|c|}
\hline \multirow[t]{2}{*}{ Dependent variable } & \multirow[t]{2}{*}{ Independent variables } & \multirow[t]{2}{*}{$R^{2}$} & \multirow[t]{2}{*}{ Adjusted $R^{2}$} & \multirow[t]{2}{*}{ B } & \multirow[t]{2}{*}{$\beta$} & \multirow[t]{2}{*}{$t$} & \multirow[t]{2}{*}{ Sig. } & \multicolumn{2}{|c|}{$95 \%$ Confidence interval for B } \\
\hline & & & & & & & & Lower bound & Upper bound \\
\hline \multirow[t]{3}{*}{ HI-MoCA score } & 4FA & 0.09 & 0.08 & -0.04 & -0.25 & -4.03 & 0.000 & -0.05 & -0.02 \\
\hline & Self-report HL & & & 1.04 & 0.18 & 2.88 & 0.004 & 0.33 & 1.75 \\
\hline & Education years & & & 0.14 & 0.15 & 2.63 & 0.009 & 0.04 & 0.24 \\
\hline \multirow[t]{3}{*}{ SWM between error } & Age & 0.09 & 0.08 & 0.76 & 0.21 & 3.69 & 0.000 & 0.36 & 1.17 \\
\hline & Social loneliness & & & -5.24 & -0.38 & -3.46 & 0.001 & -8.22 & -2.26 \\
\hline & Loneliness & & & 2.69 & 0.26 & 2.41 & 0.017 & 0.49 & 4.89 \\
\hline \multirow[t]{2}{*}{ SWM within error } & 3HFA & 0.04 & 0.04 & 0.05 & 0.17 & 2.99 & 0.003 & 0.02 & 0.08 \\
\hline & Education years & & & 0.26 & 0.14 & 2.34 & 0.020 & 0.04 & 0.48 \\
\hline \multirow[t]{3}{*}{ SWM strategy } & Social loneliness & 0.06 & 0.05 & -2.02 & -0.46 & -3.99 & 0.000 & -3.01 & -1.02 \\
\hline & Loneliness & & & 1.19 & 0.36 & 3.11 & 0.002 & 0.44 & 1.94 \\
\hline & Anxiety & & & -0.13 & -0.14 & -2.29 & 0.023 & -0.24 & -0.02 \\
\hline \multirow[t]{5}{*}{ PAL error (All shapes) } & Education years & 0.11 & 0.09 & -1.58 & -0.17 & -2.95 & 0.003 & -2.63 & -0.53 \\
\hline & Intelligent activities & & & -0.35 & -0.16 & -2.89 & 0.004 & -0.58 & -0.11 \\
\hline & Gender & & & 8.93 & 0.16 & 2.82 & 0.005 & 2.70 & 15.15 \\
\hline & Social activities & & & 1.37 & 0.14 & 2.52 & 0.012 & 0.30 & 2.45 \\
\hline & Living arrangements & & & 14.96 & 0.13 & 2.32 & 0.021 & 2.25 & 27.67 \\
\hline \multirow[t]{4}{*}{ PAL error (6 shapes) } & 4FA & 0.08 & 0.07 & 0.08 & 0.20 & 3.16 & 0.002 & 0.03 & 0.13 \\
\hline & Self-report HL & & & -2.78 & -0.16 & -2.64 & 0.009 & -4.86 & -0.71 \\
\hline & Depression & & & 0.19 & 0.13 & 2.31 & 0.021 & 0.03 & 0.36 \\
\hline & Education years & & & -0.32 & -0.12 & -2.09 & 0.038 & -0.63 & -0.02 \\
\hline \multirow[t]{5}{*}{ DMS correct percent } & 3HFA & 0.12 & 0.10 & -0.09 & -0.16 & -2.66 & 0.008 & -0.16 & -0.02 \\
\hline & Education years & & & 0.57 & 0.14 & 2.43 & 0.016 & 0.11 & 1.03 \\
\hline & Age & & & -0.31 & -0.12 & -2.02 & 0.045 & -0.62 & -0.01 \\
\hline & Anxiety & & & -0.26 & -0.12 & -2.22 & 0.027 & -0.49 & -0.03 \\
\hline & Gender & & & -2.88 & -0.12 & -2.06 & 0.040 & -5.64 & -0.13 \\
\hline
\end{tabular}

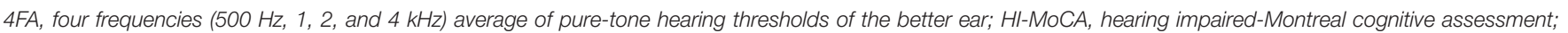

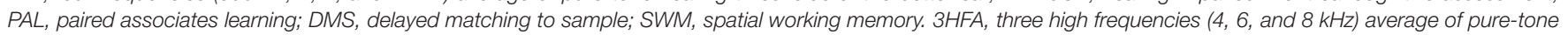
hearing thresholds of the better ear. 
Between 20 and $40 \%$ of the elderly in Western countries are reported to be lonely (Savikko et al., 2005), and the prevalence of loneliness has increased from 16 to $30 \%$ from 1992 to 2000 (Yang and Victor, 2008), possibly due to the impact of the "empty-nester" phenomenon that had impact on that (Fung et al., 2019). However, among the participants in this study, only $5.8 \%$ lived alone, the majority living with their children and even grandchildren. In China, elderly people tend to live with their children, playing an important role in caring for their grandchildren. This living arrangement is likely to have an impact on mental wellbeing and cognitive functioning (Mazzuco et al., 2017), as well as possible decreasing of loneliness and social isolation. This contrasts with the prevailing culture in Western countries, where parents tend to live alone after children move out of home, and they tend to live in residential aged-care facilities later in life; however, less than $2 \%$ of the subjects of this study population lived in aged-care centers.

\section{Clinical Implications}

This study contributes evidence to the growing global body of evidence that hearing loss and cognitive decline are significantly associated, also in Mandarin-speaking older adults. While the risk of hearing loss to cognition has not been determined, the association is something that hearing health professionals need to bear in mind when managing and counseling their patients.

\section{Limitations}

One of the limitations in this study is that the age of participants recruited generally reflected their level of hearing loss and that there were relatively fewer participants with increasing severity of hearing loss. However, this is consistent with the prevalence of hearing loss in the population in relation to age. This study was designed as a longitudinal study, so as to examine the association between ARHL and cognitive decline over time. Another potential limitation is that participant fatigue may have affected some of the more strenuous tests.

\section{Future Directions}

These findings warrant longitudinal studies to better understand the association between hearing loss and cognitive impairment over time and also to investigate how confounding factors contribute to the cognitive functions overall. Recent studies have studied the impact of hearing aids and cochlear implantation on cognitive functions of older adults with hearing loss (Jayakody et al., 2017, 2020; Kramer et al., 2018; Maharani et al., 2018). The results indicate that hearing rehabilitation has some degree of impact on improving cognitive functions. Based on the findings, we recommend a randomized control trial investigating the impact of hearing rehabilitation on the cognitive functions of Mandarin-speaking older adults.

\section{REFERENCES}

Amieva, H., Ouvrard, C., Giulioli, C., Meillon, C., Rullier, L., and Dartigues, J. F. (2015). Self-reported hearing loss, hearing aids, and cognitive decline in elderly adults: a 25-year study. J. Am. Geriatr. Soc. 63, 2099-2104. doi: 10.1111/jgs. 13649

\section{CONCLUSION}

This study provides more evidence for the association between hearing loss and cognitive impairment in Mandarin-speaking older adults. This study revealed that the hearing loss has a significant relationship with cognitive impairment in a Chinesespeaking population.

\section{DATA AVAILABILITY STATEMENT}

The original contributions presented in the study are included in the article/Supplementary Material, further inquiries can be directed to the corresponding author/s.

\section{ETHICS STATEMENT}

The studies involving human participants were reviewed and approved by the University of Western Australia-Human Research Ethics Committee, the Institutional Review Board of Beijing Tongren Hospital, Capital Medical University. The participants provided their written informed consent to participate in this study.

\section{AUTHOR CONTRIBUTIONS}

DJ and XF designed the experiments. XF carried out the experiments and wrote the manuscript. XF, BL, SW, RE, and DJ analyzed the experimental results. RE, DJ, BL, and SW reviewed the manuscript. All authors contributed to the article and approved the submitted version.

\section{FUNDING}

This research was supported by the Reform and Development Grant of Beijing Institute of Otolaryngology, Natural Science Foundation of China (Grant No. 81870715).

\section{SUPPLEMENTARY MATERIAL}

The Supplementary Material for this article can be found online at: https://www.frontiersin.org/articles/10.3389/fnins. 2021.749273/full\#supplementary-material

Balbag, M. A., Pedersen, N. L., and Gatz, M. (2014). Playing a musical instrument as a protective factor against dementia and cognitive impairment: a populationbased twin study. Int. J. Alzheimers Dis. 2014:836748. doi: 10.1155/2014/83 6748

Bidelman, G. M., Hutka, S., and Moreno, S. (2013). Tone language speakers and musicians share enhanced perceptual and cognitive abilities for musical pitch: 
evidence for bidirectionality between the domains of language and music. PLoS One 8:e60676. doi: 10.1371/journal.pone.0060676

Boss, L., Kang, D. H., and Branson, S. (2015). Loneliness and cognitive function in the older adult: a systematic review. Int. Psychoger. 27, 541-553. doi: 10.1017/ s1041610214002749

Cambridge Cognition Ltd (2014). CANTABeclipse Test Administration Guide. Cambridge: Cambridge Cognition Limited.

Campos-Magdaleno, M., Leiva, D., Pereiro, A. X., Lojo-Seoane, C., Mallo, S. C., Facal, D., et al. (2020). Changes in visual memory in mild cognitive impairment: a longitudinal study with CANTAB. Psychol. Med. 51, 2465-2475. doi: 10.1017/ s0033291720001142

Chan, R. C., Xu, T., Huang, J., Wang, Y., Zhao, Q., Shum, D. H., et al. (2012). Extending the utility of the depression anxiety stress scale by examining its psychometric properties in Chinese settings. Psychiatry Res. 200, 879-883. doi: 10.1016/j.psychres.2012.06.041

Ciorba, A., Bianchini, C., Pelucchi, S., and Pastore, A. (2012). The impact of hearing loss on the quality of life of elderly adults. Clin. Interv. Aging 7, 159-163. doi: $10.2147 /$ cia.S26059

Deal, J. A., Sharrett, A. R., Albert, M. S., Coresh, J., Mosley, T. H., Knopman, D., et al. (2015). Hearing impairment and cognitive decline: a pilot study conducted within the atherosclerosis risk in communities neurocognitive study. Am. J. Epidemiol. 181, 680-690. doi: 10.1093/aje/kwu333

Donovan, N. J., Wu, Q., Rentz, D. M., Sperling, R. A., Marshall, G. A., and Glymour, M. M. (2017). Loneliness, depression and cognitive function in older U.S. adults. Int. J. Geriatr. Psychiatry 32, 564-573. doi: 10.1002/gps. 4495

Dupuis, K., Pichora-Fuller, M. K., Chasteen, A. L., Marchuk, V., Singh, G., and Smith, S. L. (2015). Effects of hearing and vision impairments on the montreal cognitive assessment. Neuropsychol. Dev. Cogn. Section B Aging, Neuropsychol. Cogn. 22, 413-437. doi: 10.1080/13825585.2014.968084

Fung, A. W. T., Lee, A. T. C., Cheng, S. T., and Lam, L. C. W. (2019). Loneliness interacts with family relationship in relation to cognitive function in Chinese older adults. Int. Psychogeriatr. 31, 467-475. doi: 10.1017/S104161021800 1333

Gussekloo, J., de Craen, A. J., Oduber, C., van Boxtel, M. P., and Westendorp, R. G. (2005). Sensory impairment and cognitive functioning in oldest-old subjects: the leiden 85+ study. Am. J. Geriatr. Psychiatry 13, 781-786. doi: 10.1176/appi. ajgp.13.9.781

Gyanwali, B., Hilal, S., Venketasubramanian, N., Chen, C., and Loo, J. H. Y. (2020). Hearing handicap in Asian patients with dementia. Am. J. Otolaryngol. 41:102377. doi: 10.1016/j.amjoto.2019.102377

Haile, L. M., Kamenov, K., Briant, P. S., Orji, A. U., Steinmetz, J. D., Abdoli, A., et al. (2021). Hearing loss prevalence and years lived with disability, 1990-2019: findings from the global burden of disease study 2019. Lancet 397, 996-1009. doi: 10.1016/S0140-6736(21)00516-X

Hicks, E. B., Ahsan, N., Bhandari, A., Ghazala, Z., Wang, W., Pollock, B. G., et al. (2020). Associations of visual paired associative learning task with global cognition and its potential usefulness as a screening tool for Alzheimer's dementia. Int. Psychogeriatr. doi: 10.1017/s1041610220003841 [Epub ahead of print].

Humes, L. E. (2019). The world health organization's hearing-impairment grading system: an evaluation for unaided communication in age-related hearing loss. Int. J. Audiol. 58, 12-20. doi: 10.1080/14992027.2018.1518598

Jayakody, D. M. P., Almeida, O. P., Ford, A. H., Atlas, M. D., Lautenschlager, N. T., Friedland, P. L., et al. (2020). Hearing aids to support cognitive functions of older adults at risk of dementia: the HearCog trial- clinical protocols. BMC Geriatr. 20:508. doi: 10.1186/s12877-020-01912-1

Jayakody, D. M. P., Almeida, O. P., Speelman, C. P., Bennett, R. J., Moyle, T. C., Yiannos, J. M., et al. (2018a). Association between speech and high-frequency hearing loss and depression, anxiety and stress in older adults. Maturitas 110, 86-91. doi: 10.1016/j.maturitas.2018.02.002

Jayakody, D. M. P., Friedland, P. L., Eikelboom, R. H., Martins, R. N., and Sohrabi, H. R. (2018b). A novel study on association between untreated hearing loss and cognitive functions of older adults: baseline non-verbal cognitive assessment results. Clin. Otolaryngol. 43, 182-191. doi: 10.1111/coa.12937

Jayakody, D. M. P., Friedland, P. L., Nel, E., Martins, R. N., Atlas, M. D., and Sohrabi, H. R. (2017). Impact of cochlear implantation on cognitive functions of older adults: pilot test results. Otol. Neurotol. 38, e289-e295. doi: 10.1097/ mao.0000000000001502

Kramer, S., Vasil, K. J., Adunka, O. F., Pisoni, D. B., and Moberly, A. C. (2018). Cognitive functions in adult cochlear implant users, cochlear implant candidates, and normal-hearing listeners. Laryngoscope Investig Otolaryngol. 3 , 304-310. doi: 10.1002/lio2.172

Lara, E., Caballero, F. F., Rico-Uribe, L. A., Olaya, B., Haro, J. M., Ayuso-Mateos, J. L., et al. (2019). Are loneliness and social isolation associated with cognitive decline? Int. J. Geriatr. Psychiatry 34, 1613-1622. doi: 10.1002/gps.5174

Leung, G. T., de Jong Gierveld, J., and Lam, L. C. (2008). Validation of the Chinese translation of the 6-item de jong gierveld loneliness scale in elderly Chinese. Int. Psychogeriatr. 20, 1262-1272. doi: 10.1017/s1041610208007552

Leung, G. T., Fung, A. W., Tam, C. W., Lui, V. W., Chiu, H. F., Chan, W. M., et al. (2010). Examining the association between participation in late-life leisure activities and cognitive function in community-dwelling elderly Chinese in hong kong. Int. Psychogeriatr. 22, 2-13. doi: 10.1017/S1041610209991025

Lin, F. R., Metter, E. J., O'Brien, R. J., Resnick, S. M., Zonderman, A. B., and Ferrucci, L. (2011). Hearing loss and incident dementia. Arch. Neurol. 68, 214-220. doi: 10.1001/archneurol.2010.362

Lin, V. Y. W., Chung, J., Callahan, B. L., Smith, L., Gritters, N., Chen, J. M., et al. (2017). Development of cognitive screening test for the severely hearing impaired: hearing-impaired MoCA. Laryngoscope 127, S4-S11. doi: 10.1002/ lary. 26590

Livingston, G., Sommerlad, A., Orgeta, V., Costafreda, S. G., Huntley, J., Ames, D., et al. (2017). Dementia prevention, intervention, and care. Lancet 390, 2673-2734. doi: 10.1016/s0140-6736(17)31363-6

Lovibond, P. F., and Lovibond, S. H. (1995). The structure of negative emotional states: comparison of the depression anxiety stress scales (DASS) with the beck depression and anxiety inventories. Behav. Res. Ther. 33, 335-343. doi: 10.1016/0005-7967(94)00075-u

Luo, Y., He, P., Guo, C., Chen, G., Li, N., and Zheng, X. (2018). Association between sensory impairment and dementia in older adults: evidence from China. J. Am. Geriatr. Soc. 66, 480-486. doi: 10.1111/jgs.15202

Maharani, A., Dawes, P., Nazroo, J., Tampubolon, G., and Pendleton, N. (2018). Longitudinal relationship between hearing aid use and cognitive function in older americans. J. Am. Geriatr. Soc. 66, 1130-1136. doi: 10.1111/jgs.15363

Mansky, R., Marzel, A., Orav, E. J., Chocano-Bedoya, P. O., Grünheid, P., Mattle, M., et al. (2020). Playing a musical instrument is associated with slower cognitive decline in community-dwelling older adults. Aging Clin. Exp. Res. 32, 1577-1584. doi: 10.1007/s40520-020-01472-9

Mazzuco, S., Meggiolaro, S., Ongaro, F., and Toffolutti, V. (2017). Living arrangement and cognitive decline among older people in Europe. Ageing Soc. 37, 1111-1133. doi: 10.1017/S0144686X16000374

Nasreddine, Z. S., Phillips, N. A., Bédirian, V., Charbonneau, S., Whitehead, V., Collin, I., et al. (2005). The montreal cognitive assessment, MoCA: a brief screening tool for mild cognitive impairment. J. Am. Geriatr. Soc. 53, 695-699. doi: 10.1111/j.1532-5415.2005.53221.x

Ngo, M. K., Vu, K. P., and Strybel, T. Z. (2016). Effects of music and tonal language experience on relative pitch performance. Am. J. Psychol. 129, 125-134. doi: 10.5406/ameripsyc.129.2.0125

Olsen, R. K., Nichols, E. A., Chen, J., Hunt, J. F., Glover, G. H., Gabrieli, J. D., et al. (2009). Performance-related sustained and anticipatory activity in human medial temporal lobe during delayed match-to-sample. J. Neurosci. 29, 1188011890. doi: 10.1523/jneurosci.2245-09.2009

Ren, F., Luo, J., Ma, W., Xin, Q., Xu, L., Fan, Z., et al. (2019). Hearing loss and cognition among older adults in a Han Chinese cohort. Front. Neurosci. 13:632. doi: $10.3389 /$ fnins. 2019.00632

Salvi, R., Ding, D., Jiang, H., Chen, G. D., Greco, A., Manohar, S., et al. (2018). Hidden age-related hearing loss and hearing disorders: current knowledge and future directions. Hearing Balance Commun. 16, 74-82. doi: 10.1080/21695717. 2018.1442282

Savikko, N., Routasalo, P., Tilvis, R. S., Strandberg, T. E., and Pitkälä, K. H. (2005). Predictors and subjective causes of loneliness in an aged population. Arch. Gerontol. Geriatr. 41, 223-233. doi: 10.1016/j.archger.2005.03.002

Su, P., Hsu, C. C., Lin, H. C., Huang, W. S., Yang, T. L., Hsu, W. T., et al. (2017). Age-related hearing loss and dementia: a 10-year national population-based study. Eur. Arch. Otorhinol. 274, 2327-2334. doi: 10.1007/s00405-017-4471-5 
Verghese, J., Lipton, R. B., Katz, M. J., Hall, C. B., Derby, C. A., Kuslansky, G., et al. (2003). Leisure activities and the risk of dementia in the elderly. N. Engl. J. Med. 348, 2508-2516. doi: 10.1056/NEJMoa022252

Wang, K., Shi, H. S., Geng, F. L., Zou, L. Q., Tan, S. P., Wang, Y., et al. (2016). Crosscultural validation of the depression anxiety stress scale-21 in China. Psychol Assess 28, e88-e100. doi: 10.1037/pas0000207

Wang, P., Li, J., Li, H. J., Huo, L., and Li, R. (2016). Mild cognitive impairment is not "Mild" at all in altered activation of episodic memory brain networks: evidence from ale meta-analysis. Front Aging Neurosci 8:260. doi: 10.3389/fnagi. 2016.00260

Yang, F., Zhang, J., and Wang, J. (2018). Correlates of loneliness in older adults in shanghai, China: does age matter? BMC Geriatr. 18:300. doi: 10.1186/s12877018-0994-x

Yang, K., and Victor, C. (2008). The prevalence of and risk factors for loneliness among older people in China. Ageing Soc. 28, 305-327. doi: 10.1017/ S0144686X07006848

Zahodne, L. B., Stern, Y., and Manly, J. J. (2015). Differing effects of education on cognitive decline in diverse elders with low versus high educational attainment. Neuropsychology 29, 649-657. doi: 10.1037/neu0000141
Conflict of Interest: The authors declare that the research was conducted in the absence of any commercial or financial relationships that could be construed as a potential conflict of interest.

The editor KL has declared a shared institutional affiliation with the authors XF, $\mathrm{BL}$, and $\mathrm{SW}$ at the time of review.

Publisher's Note: All claims expressed in this article are solely those of the authors and do not necessarily represent those of their affiliated organizations, or those of the publisher, the editors and the reviewers. Any product that may be evaluated in this article, or claim that may be made by its manufacturer, is not guaranteed or endorsed by the publisher.

Copyright (c) $2021 \mathrm{Fu}$, Liu, Wang, Eikelboom and Jayakody. This is an open-access article distributed under the terms of the Creative Commons Attribution License (CC BY). The use, distribution or reproduction in other forums is permitted, provided the original author(s) and the copyright owner(s) are credited and that the original publication in this journal is cited, in accordance with accepted academic practice. No use, distribution or reproduction is permitted which does not comply with these terms. 